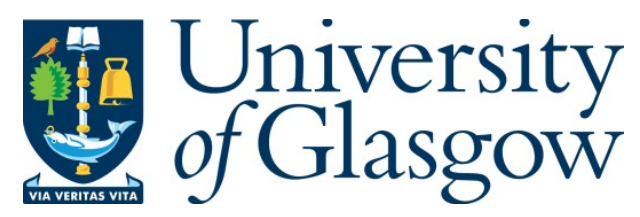

Kivisaari, P., Sadi, T., Oksanen, J., and Tulkki, J. (2016) Monte Carlo study of nonquasiequilibrium carrier dynamics in III-N LEDs. Optical and Quantum

Electronics, 48(2), 154.

There may be differences between this version and the published version. You are advised to consult the publisher's version if you wish to cite from it.

http://eprints.gla.ac.uk/129954/

Deposited on: 28 November 2016

Enlighten - Research publications by members of the University of Glasgow http://eprints.gla.ac.uk 


\title{
Monte Carlo study of non-quasiequilibrium carrier dynamics in III-N LEDs
}

\author{
Pyry Kivisaari · Toufik Sadi · Jani Oksanen · \\ Jukka Tulkki
}

Received: date / Accepted: date

\begin{abstract}
Hot carrier effects have been observed in recent measurements of IIINitride (III-N) light-emitting diodes (LEDs). In this paper we carry out bipolar Monte Carlo (MC) simulations for electrons and holes in a typical III-N multi-quantum well (MQW) LED. According to our simulations, significant non-quasiequilibrium carrier distributions exist in the barrier layers of the structure. This is observed as average carrier energies much larger than the $1.5 k_{B} T$ corresponding to quasi-equilibrium. Due to the small potential drop over the MQW being modest, the non-quasiequilibrium carriers can be predominantly ascribed to nnp and npp Auger processes taking place in the QWs. Further investigations are needed to determine the effects of hot carriers on the macroscopic device characteristics of real devices.
\end{abstract}

Keywords III-Nitride LEDs · Monte Carlo simulations · Hot carriers · Nonequilibrium hole distribution

\section{Introduction}

The basic operation of III-Nitride (III-N) multi-quantum well (MQW) light-emitting diodes (LEDs) is well-known, and the related technology is reaching maturity [1]. The large efforts over the last ten years to resolve the cause of the efficiency droop are also finally bearing fruit, ruling out leakage current as a major cause [2]. The next developments on III-N LEDs will therefore require using unconventional device

P. Kivisaari

Division of Solid State Physics, Lund University, Sweden

E-mail: pyry.kivisaari@ftf.lth.se

T. Sadi

School of Engineering, University of Glasgow, UK

J. Oksanen and J. Tulkki

School of Science, Aalto University, Finland 
structures and better substrates, as well as developing significantly improved modeling tools. On the modeling side, hot carriers constitute a mechanism that is largely ignored in conventional simulation tools.

Recent measurements have, however, revealed a presence of hot carriers in conventional III-N MQW LED structures [3,4]. In their work [3], Iveland et al. measured electron energy spectra from a cesiated p-type contact of an LED under forward bias, and they observed a clear separate high-energy peak. Binder et al., on the other hand, measured photoluminescence from a dual-wavelength structure (greenand UV-emitting quantum wells) and observed UV luminescence, whose wavelength was significantly smaller than that of the pump laser. As direct laser excitation of the UV quantum well (QW) was thereby ruled out, they concluded that the UV luminescence must result from hot carrier transport from the green QWs to the UV QWs [4]. Note that Ref. 4 also therefore suggests a presence of hot holes in the structure. In both Refs. [3,4], the authors came to a conclusion that the hot carriers observed were caused by Auger processes. However, neither of these references can give information on the details of the hot-carrier transport and its possible effects on the device performance. On the modeling front, the commonly used drift-diffusion (DD) model cannot be used to study such hot-carrier effects, as it only accounts for quasi-equilibrium carrier distributions.

Semiempirical models have been published for inclusion of hot carriers in LED models. Based on such models, different works have suggested that hot carriers could improve carrier spreading [5] and increase leakage current through Auger excitation [6]. On the other hand, Bertazzi et al. performed Monte Carlo (MC) simulations in $\mathrm{GaN}$ and provided alternative explanations for the measured hot-carrier effects [7]. To study the hot carrier effects in realistic full LED devices directly, we have recently reported device-level MC simulations of electrons [8-10]. To account directly for both hot electrons and hot holes, more recently we have introduced a full bipolar MC simulator [11]. Our device-level MC simulations have showed, e.g., that a population of hot sidevalley electrons can exist at a p-type contact of a biased LED [8,9], and that high bias voltages may result in notable differences between DD and MC [10].

To lay the groundwork for studying the device-level effects of hot electrons and hot holes using direct MC simulations, in this work we carry out bipolar MC simulations for a III-N LED device, which has three $3 \mathrm{~nm}$ thick QWs and an EBL that is $40 \mathrm{~nm}$ thick. In particular and in contrast to previous works, in this paper we provide information on the carrier energies from full bipolar MC simulations, which also account for the non-quasiequilibrium hole distributions. We study the electron and hole densities resulting from MC simulations at a modest bias voltage. We compare the carrier densities to the average carrier energies to investigate hot carrier transport and to study where the carrier distributions are driven out of quasi-equilibrium. Our results provide insight to the extent of hot carrier effects in LEDs and also lay the groundwork for more detailed future investigations of the device-level effects. 


\section{Theory}

Majority of LED device simulations deploy the drift-diffusion (DD) model, that can also be extended for special effects such as quantum-confined Stark effect or random alloy fluctuations [12-15]. Quasi-equilibrium carrier distributions are assumed in the DD model, and hot carrier effects can be included only phenomenologically $[5,6]$. In this paper we use the ensemble Monte Carlo method, in which the semiclassical Boltzmann transport equation is solved by direct simulation of a large number of electrons and holes [16-18]. The most important equations for our MC simulation of LEDs are found in Ref. [10]. Electrostatic potential is updated during the simulation using the time-dependent electron and hole densities from the MC simulation. The simulation is run until convergence for a given bias voltage, and the carrier distribution functions are calculated as time averages from the steady-state MC simulations.

Scattering rates are needed to calculate the free-flight times and self-scattering rates. They are calculated for transitions between different Bloch states by using Fermi's golden rule with the scattering process specific Hamiltonians, as explained in Ref. [19]. The final states of the carriers after scattering are finally given by the selection rules pertaining to the specific scattering process. Intraband scattering processes included in the simulations are the deformation potential and electrostatic acoustic and optical phonon scattering, electron-electron and electron-hole scattering, ionized impurity scattering (important in doped regions), and alloy disorder scattering in ternary alloys. Surface roughness scattering is considered at material interfaces. Interband scattering, or recombination, is calculated from the time-dependent electron and hole densities using the semiempirical models detailed in [20].

\section{Results and discussion}

Bipolar MC simulation results are carried out for a III-N LED with n-type GaN, a MQW region of three QWs, an EBL, and a p-type GaN. The In composition is 0.18 in the QWs, and the $\mathrm{Al}$ composition is 0.15 in the EBL. Ionized doping densities are $10^{24} \mathrm{~m}^{-3}$ for both acceptors and donors.

Figure 1(a) shows the MC electron and hole densities as a function of position in the LED at a bias voltage of $3.55 \mathrm{~V}$ and current density of $2.78 \mathrm{~A} / \mathrm{cm}^{2}$. Electron density is equal to the ionized donor density in the n-side, and hole density is likewise equal to the ionized acceptor density in the p-side. In the MQW region, the electron density is high in the QWs and smaller in the barrier layers. Electron density in the EBL and p-type GaN is small, indicating also a small electron leakage. Hole density, on the other hand, is much smaller than electron density in the barrier layers between the QWs. This can be expected, as the mobility of holes is significantly smaller than that of electrons (in MC simulations corresponding to faster average relaxation rates). Conventional DD simulations typically exhibit large quasi-Fermi losses for holes in the barrier layers (see, e.g., band diagrams in Refs. [2,15]). Studying the small MC hole densities in the barrier layers shown in Fig. 1(a) might give further insight to these losses in future works. 


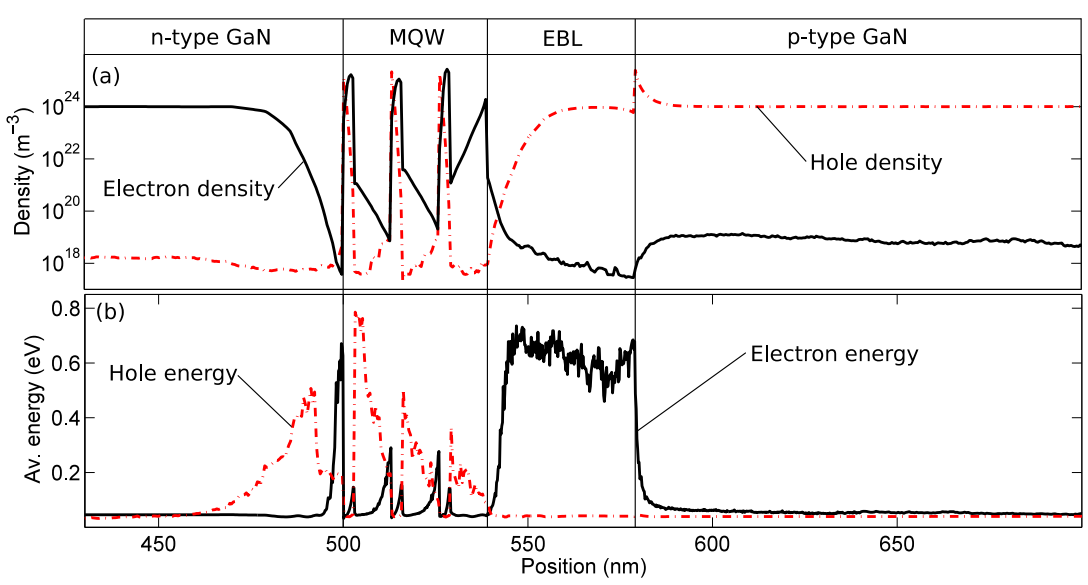

Fig. 1 (a) Electron and hole densities as a function of position in the LED. The bias voltage is $3.55 \mathrm{~V}$, and the current density is $2.78 \mathrm{~A} / \mathrm{cm}^{2}$. (b) Electron and hole average energies as a function of position in the LED. The bias voltage is $3.55 \mathrm{~V}$, and the current density is $2.78 \mathrm{~A} / \mathrm{cm}^{2}$.

Figure 1(b) shows the electron and hole average energies measured from the band edges as a function of position. Note that the average energy includes both quasiequilibrium carriers and hot carriers. In regions with a small carrier density, even a small number of hot carriers increases the average carrier energy significantly. Due to Auger excitation, hot carriers can have roughly $2.5 \mathrm{eV}$ higher energy than the quasi-equilibrium carriers. Therefore hot carriers cause a notable increase the average energy especially in regions with small carrier densities. To understand the complete picture, more detailed studies of the full distribution functions will, however, be required. In Fig. 1(b), the average electron energy is roughly equal to the quasi-equilibrium value of $1.5 k_{B} T$ (corresponding to the average kinetic energy in Boltzmann statistics) in the n-type GaN on the left, and it stays fairly close to that even in the MQW region. The largest deviation within the MQW region takes place in the $\mathrm{n}-\mathrm{GaN}$ just before the first QW, most probably due to the small density of quasiequilibrium electrons and a large number of Auger-excited electrons there. Over the whole EBL, the electron energy is significantly larger than $1.5 k_{B} T$. This indicates that a significant part of electrons flowing over the EBL is due to hot electrons. Moreover, as the potential drop over the LED is only roughly $0.25 \mathrm{eV}$, the elevated average electron energy of $0.7 \mathrm{eV}$ in the EBL is heavily contributed to by Auger-excited electrons. On the other hand, the three smallest peaks of the electron energy correspond to the QWs. They suggest that even the QW electron distributions are slightly out of quasiequilibrium despite the large electron density. However, quantum-mechanical models are needed for more detailed studies of the QW carrier distributions.

Hole energy in Fig. 1(b), on the other hand, is roughly equal to the quasi-equilibrium value of $1.5 k_{B} T$ in the p-type GaN and also in the EBL. In contrast, in the MQW region the hole energy has large peaks in the barrier layers. Again, as the potential drop over the MQW region is small, the elevated hole energy is mostly resulting from the Auger-excited holes. To gain further insight on the hole energy distribution, we can compare it with the hole density shown in Fig. 1(a). The QWs have 


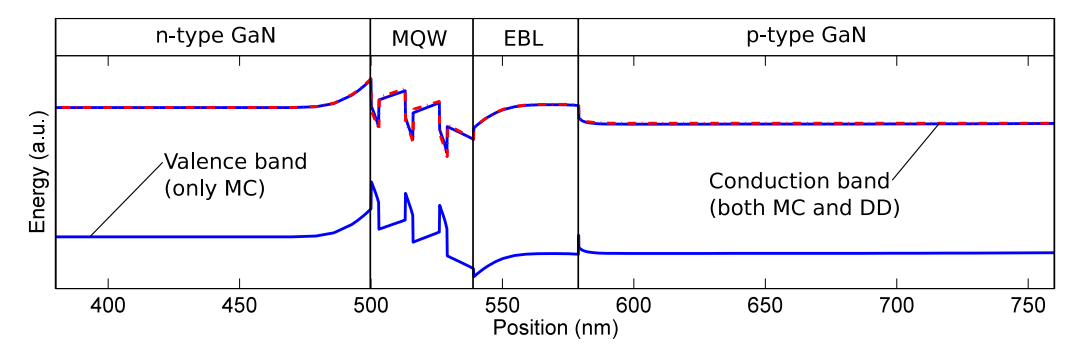

Fig. 2 Scaled band diagram as a function of position in the LED. The applied voltage is $3.55 \mathrm{~V}$, and the current density is $2.78 \mathrm{~A} / \mathrm{cm}^{2}$. Conduction band is shown resulting from both MC and DD simulations.

large hole densities, and comparison to Fig. 1(b) shows that they follow roughly their quasi-equilibrium distribution, corresponding to the average energy of $1.5 k_{B} T$. The barriers, in contrast, have very small hole densities (Fig. 1(a)), and comparison to the hole energy shown in Fig. 1(b) suggests that hole transport over the barriers is significantly contributed to by hot holes. Figure 1(b) shows that some of the highenergy holes even reach the n-type $\mathrm{GaN}$, where the hole distribution relaxes to its quasi-equilibrium distribution within roughly $50 \mathrm{~nm}$.

Furthermore, in Fig. 1(b) the maximum average energy is ca. $0.7 \mathrm{eV}$ for both electrons and holes. Assuming the extreme case that all the hot carriers have received an energy of $E_{g} \sim 2.5 \mathrm{eV}$ while thermalized carriers only have an energy of $1.5 k_{B} T \sim$ $40 \mathrm{meV}$ then suggests that at least $1 / 4$ of the carriers out of quasi-equilibrium are Auger-excited. In reality the fraction of hot carriers is expected to be even larger, as the average energy of hot carriers is smaller than $E_{g}$ due to ongoing relaxation.

The conduction and valence band edges are shown in Fig. 2 at the bias voltage of $3.55 \mathrm{~V}$. Conduction band is also shown as resulting from the DD simulation, and it is very close to the $\mathrm{MC}$ result. The EBL forms a large potential barrier for electrons, and in the MC simulations a large part of electrons crossing the EBL are Auger-excited electrons, as demonstrated by the large electron average energy in the EBL (Fig. 1(b)). Holes experience much faster intraband scattering than electrons, and therefore they lose their energy faster and over considerably shorter distances than electrons. This results in a smaller fraction of holes in the QWs and barriers, which makes crossing the GaN barriers less efficient for holes than for electrons. Comparing with Fig. 1(b), it can be seen that in MC simulations Auger-generated hot holes constitute a major part of hole transport over the GaN barriers. On the other hand, the band diagram shows that the electrostatic potential is very similar between the DD (quasiequilibrium) and MC (non-quasiequilibrium) simulations. The small difference between MC and DD indicates that in the simulated structure, the electrostatic potential profile is largely still determined by the carriers that are in quasi-equilibrium. Further investigations are needed to clearly separate the effects of quasi-equilibrium and non-quasiequilibrium carrier distributions in typical III-N LEDs. 


\section{Conclusions}

We carried out bipolar MC simulations for electrons and holes in a III-Nitride LED structure with three InGaN QWs and an AlGaN EBL. According to our simulations, significant non-quasiequilibrium carrier distributions exist in the barrier layers. This was observed as average carrier energies much larger than the $1.5 k_{B} T$ corresponding to quasi-equilibrium. In the simulations the potential drop over the MQW was modest, and therefore the non-quasiequilibrium carriers were mostly caused by nnp and npp Auger processes. However, the band diagram was similar between MC and a corresponding DD simulation, indicating that the electrostatic potential profile was mostly contributed to by quasi-equilibrium carriers. Further investigations are needed to determine the effects of hot carriers on the device characteristics of real devices.

Acknowledgements We acknowledge the financial support from the Nokia Foundation, the Finnish Cultural Foundation, and the Aalto Energy Efficiency Research Programme.

\section{References}

1. Nakamura, S. and Krames, M.: History of Gallium-Nitride-Based Light-Emitting Diodes for Illumination. Proc. IEEE 101, 2211-2220 (2013).

2. Piprek, J.: How to decide between competing efficiency droop models for GaN-based light-emitting diodes. Appl. Phys. Lett. 107, 031101 (2015)

3. Iveland, J., Piccardo, M., Martinelli, L., Peretti, J., Choi, J. W., Young, N., Nakamura, S., Speck, J. S., and Weisbuch, C.: Origin of electrons emitted into vacuum from InGaN light emitting diodes. Appl. Phys. Lett. 105, 052103 (2014)

4. Binder, M., Nirschl, A., Zeisel, R., Hager, T., Lugauer, H.-J., Sabathil, M., Bougeard, D., Wagner, J., and Galler, B.: Identification of nnp and npp Auger recombination as significant contributor to the efficiency droop in (GaIn)N quantum wells by visualization of hot carriers in photoluminescence. Appl. Phys. Lett. 103, 071108 (2013)

5. Li, Z. M. S.: Non-local transport in numerical simulation of GaN LED. J. Comput. Electron. 14, 409415 (2015).

6. Deppner, M., Römer, F., and Witzigmann, B.: Auger carrier leakage in III-nitride quantum-well light emitting diodes. Phys. Status Solidi RRL 6, 418-420 (2012).

7. Bertazzi, F., Goano, M., Zhou, X., Calciati, M., Ghione, G., Matsubara, M., and Bellotti, E.: Looking for Auger signatures in III-nitride light emitters: A full-band Monte Carlo perspective. Appl. Phys. Lett. 106, 061112 (2015).

8. Sadi, T., Kivisaari, P., Oksanen, J., and Tulkki, J.: On the correlation of the Auger generated hot electron emission and efficiency droop in III-N light-emitting diodes. Appl. Phys. Lett. 105, 091106 (2014)

9. Sadi, T., Kivisaari, P., Oksanen, J., and Tulkki, J.: Microscopic simulation of hot electron transport in III-N light-emitting diodes. Opt. Quant. Electron. 47, 1509-1518 (2015)

10. Kivisaari, P., Oksanen, J., Tulkki, J., and Sadi, T.: Monte Carlo simulation of hot carrier transport in III-N LEDs. J. Comput. Electron. 14, 382-397 (2015)

11. Kivisaari, P., Sadi, T., Oksanen, J., and Tulkki, J.: Bipolar Monte Carlo simulation of electrons and holes in III-N LEDs. Proc. SPIE 9363, 93631S (2015).

12. Kivisaari, P., Oksanen, J., and Tulkki, J.: Polarization doping and the efficiency of III-nitride optoelectronic devices. Appl. Phys. Lett. 103, 211118 (2013)

13. Piprek, J., Römer, F., and Witzigmann, B.: On the uncertainty of the Auger recombination coefficient extracted from InGaN/GaN light-emitting diode efficiency droop measurements. Appl. Phys. Lett. 106, $101101(2015)$

14. Yang, T.-J., Shivaraman, R., Speck, J. S., and Wu, Y.-R.: The influence of random indium alloy fluctuations in indium gallium nitride quantum wells on the device behavior. J. Appl. Phys. 116, 113104 (2014). 
15. Römer, F. and Witzigmann, B.: Acceptor impurity activation in III-nitride light emitting diodes. Appl. Phys. Lett. 106, 021107 (2015).

16. Jacoboni, C. and Reggiani, L.: The Monte Carlo method for the solution of charge transport in semiconductors with applications to covalent materials. Rev. Mod. Phys. 55, 645 (1983).

17. Sadi, T., Kelsall, R. W., and Pilgrim, N. J.: Investigation of Self-Heating Effects in Submicrometer GaN/AlGaN HEMTs Using an Electrothermal Monte Carlo Method. IEEE Trans. Electr. Dev. 53, 28922900 (2006)

18. Sadi, T. and Kelsall, R. W.: Hot-Phonon Effect on the Electrothermal Behavior of Submicrometer III-V HEMTs. IEEE Electr. Dev. Lett. 28, 787-789 (2007).

19. Ridley, B. K.: Quantum Processes in Semiconductors. Clarendon Press, Oxford (1999).

20. Heikkilä, O., Oksanen, J., and Tulkki, J.: Ultimate limit and temperature dependency of light-emitting diode efficiency. J. Appl. Phys. 105, 093119 (2009). 